\title{
An Open-Source 3D Printed Underactuated Robotic Gripper
}

\author{
Yedige Tlegenov, Kuat Telegenov, Almas Shintemirov \\ Department of Robotics and Mechatronics \\ Nazarbayev University \\ Astana, Kazakhstan, 010000 \\ Emails: yedige.tlegenov@nu.edu.kz, \\ ktelegenov@nu.edu.kz, ashintemirov@nu.edu.kz
}

\begin{abstract}
In this paper authors present an open source lowcost basic robotic end effector platform for facilitating research on robotic grasping. The 3D design model of a three fingered underactuated robotic gripper is presented and manufactured with minimal number of $3 \mathrm{D}$ printed components and an off-theshelf servomotor actuator. An underactuated finger, gear train mechanisms and an overall gripper assembly design are described in details followed by illustration and discussion of grasping of objects with various geometries. The presented open source gripper design will be released for downloading on the authors' research lab web-site www. alaris.kz and can be useful for robotic researchers as a platform to build their own robotic end effector solutions for research and educational purposes.
\end{abstract}

\section{INTRODUCTION}

Development of robotic end effectors that are employed for grasping of a variety of objects is an active research area. Various robotic end effectors were developed for wide range of applications where reproducing the human hand functionality is required [1]-[3]. However, in most of industrial and service applications manipulation of objects with anthropomorphic robotic hands is not required and three fingered grasping devices are more sufficient [4]. Considering mechanical finger designs, a lot of robotic grippers utilize individual actuation of each joint of the finger using a small high precision DC servomotors [5], [6]. This provides high number of controllable degrees of freedom (DOF), which is very suitable for grasping of complex shape objects. On the other hand, usage of multiple actuators in each finger mechanism results in high cost of the device and control complexity.

A number of designs utilize pulley/tendon driven mechanisms for wide range of robotics systems [7]. They have many advantages such as low weight, less number of actuators and high degree of adaptability. Among disadvantages are limitations in load carrying capacity and low wear resistance. Therefore, as an alternative, mechanical linkage systems are utilized for different designs of underactuated artificial fingers [8], [9].

There are many simple designs of commercially available grippers for grasping cylindrical and spherical shape objects. For example, a 3-fingered concentric gripper [10] is designed for relatively high payload industrial applications, but it is not able to perform enveloping grasping. The reconfigurable gripper [11] with convex shape thin fingers is designed for picking round objects and placing them in a tray with little space for movement.

In general, many of the commercially available hands do not accommodate extensive customisation of the design features for attachment to different robotic arm platforms or integration of additional sensors for research purposes [12]. To address such problems 3D printing rapid prototyping technology is actively applied for manufacturing of low-cost robotic hands [13], [14]. Aiming to provide a basic robotic platform with minimal number of 3D printed components and off-the-shelf actuator for facilitating robotic research efforts, a 3D printed open source tendon driven hand is proposed in [12]. State-ofthe-art of the grasping in robotics recently presented in [15]. To extend the choice of robotic end effectors freely available to researchers, in this paper the authors utilize a linkage based finger system and present an open source 3D printed underactuated three fingered robotic gripper with a simple design and relatively higher payload property comparing to similar size tendon driven mechanisms. The 3D model of the gripper is created using the SolidWorks CAD software and will be available for downloading from www. alaris.kz/.

The organization of the paper is as follows. Section II describes the design and manufacturing of the $3 \mathrm{D}$ printed three-fingered robotic gripper. The grasping performance of the robotic gripper prototype is presented in Section III, which is followed by the conclusions and future work in Section IV.

\section{Design AND FABRicAtion of 3D PRINTED Three FINGERED ROBOTIC GRIPPER}

\section{A. Underactuated Finger Mechanism}

Underactuated fingers have less number of actuators than total DOFs and are widely utilized in design of various robotic hands for industrial [16] and service robotics [17]. This is largely attributed to the relatively simple design of such mechanisms comparing with fully actuated dexterous artificial fingers. At the same time, an underactuated finger mechanism should ensure close wrapping of different shape objects due to its adaptive grasping capability with one DOF actuation.

In this work, the underactuated linkage mechanism is employed for finger design described in [18], [19]. This design ensures a simple low-cost mechanical system which was one of the principles for finger design requirements [20]. 

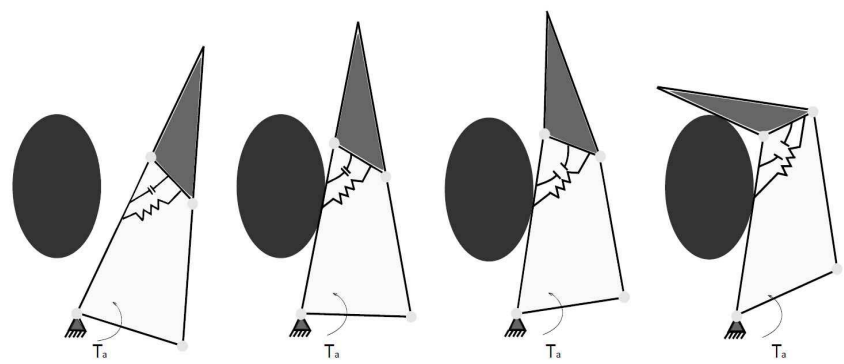

Fig. 1. Closing sequence of a 2-DOF underactuated finger.

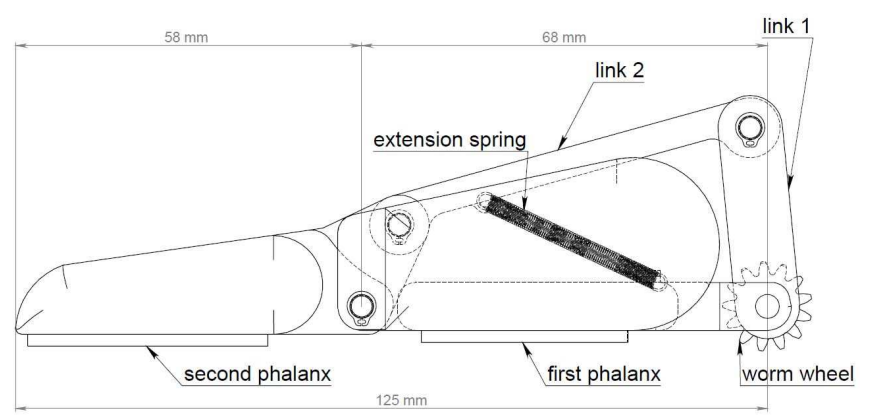

(a)

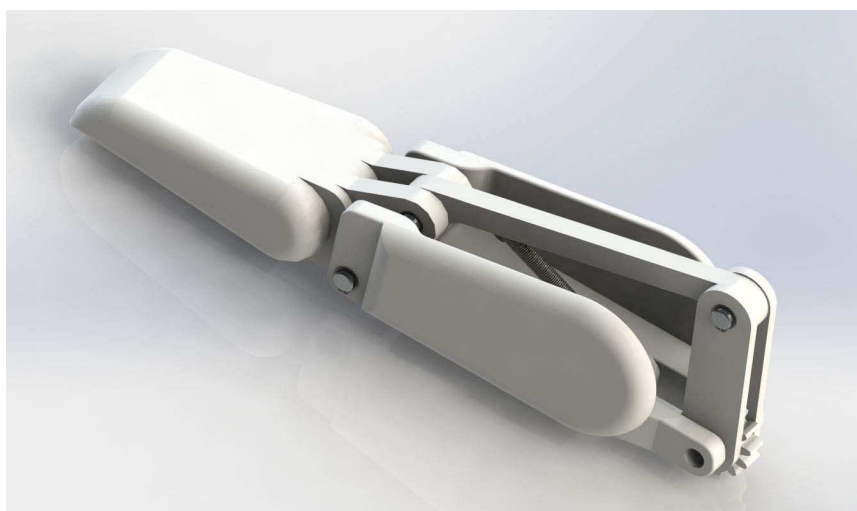

(b)

Fig. 2. 2D (a) and 3D (b) designs of the underactuated finger.

Consider a 2-DOF and one degree-of-actuation (1-DOA) finger mechanism shown in Fig.1. A passive element, i.e. a spring between the first and second phalanges is used for providing actuation of the second DOF of the finger [19]. The closing sequence of the underactuated finger is described as follows. Firstly, the finger moves as a rigid body from its initial position since no external force is acting on it. When the first phalanx of the finger is in contact with an object, the second phalanx starts movement around a pivot point to complete a full wrapping. Same method can be applied to n-phalanx finger [19].

Utilizing this principle, an underactuated finger mechanism is designed and presented in Fig. 2. The finger consists of two phalanges, two links, an extension spring and a worm wheel. Note that the worm wheel and link 1 are rigidly connected. The worm wheel transmits rotary motion to link 1 around its

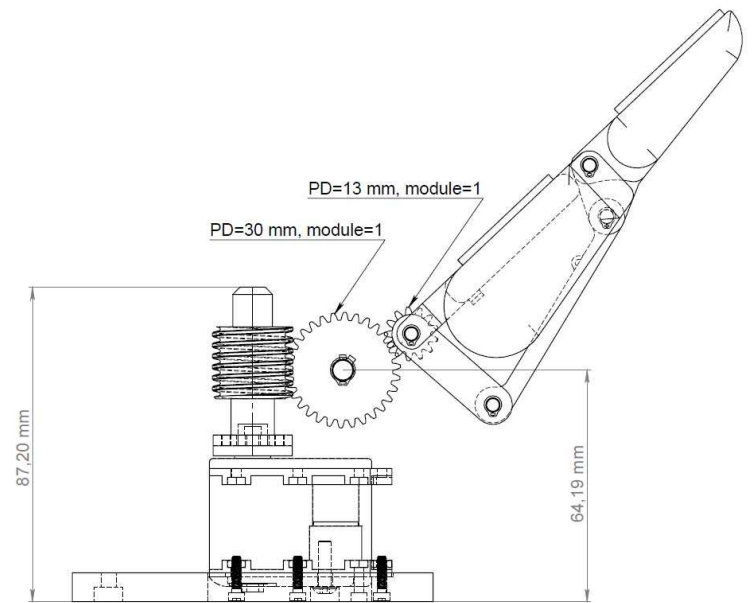

(a)

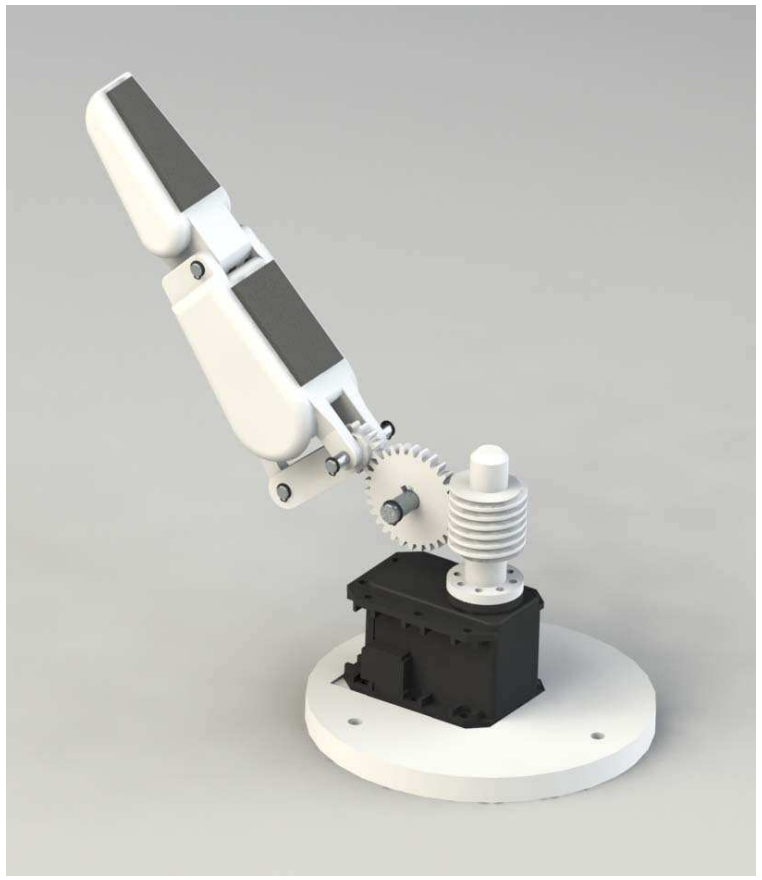

(b)

Fig. 3. 2D (a) and 3D (b) sketches of the gear train design for one finger.

pivot point; subsequently link 1 transfers the motion to link 2 .

The extension spring allows the finger to behave as a single rigid body during rotary motion around the fixed pivot. When the first phalanx touches an object, the force produced by an actuator extends the spring which starts transferring motion to the second phalanx only. Finally, both the phalanges contact an object that concludes a finger closing sequence.

Grasping characteristics of the gripper can be modified by setting various geometries of two actuation links of the fingers. For instance, changing the length of two actuation links of the finger can result in various dynamic outputs of the first and second phalanges. The surfaces of the finger components are flat so that the printer support material can be removed 


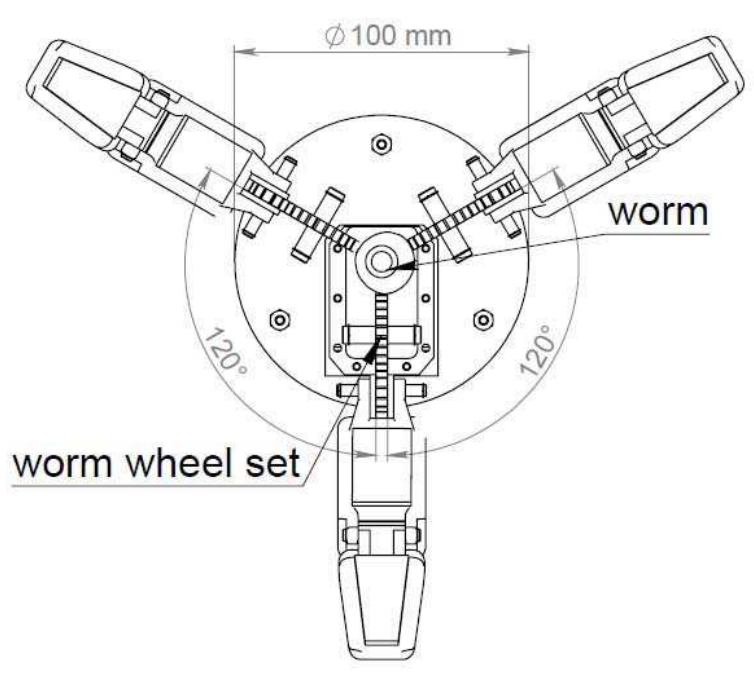

(a)

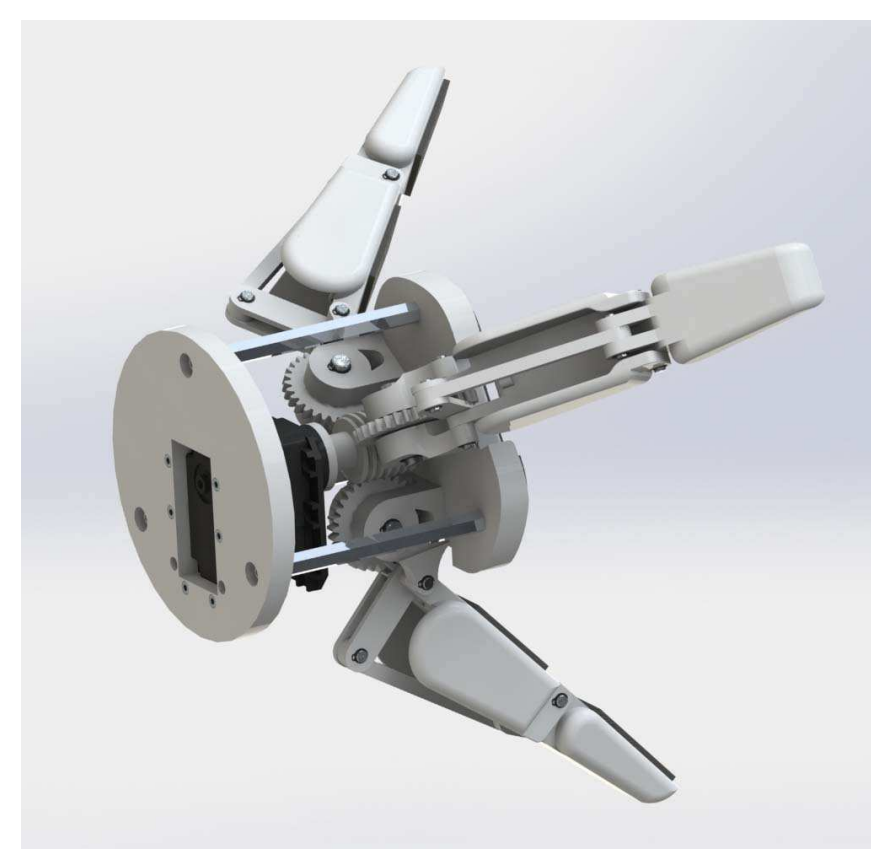

(b)

Fig. 4. Arrangement of worm wheels connected to the actuating worm in 2D (a) and 3D (b) views.

manually with little effort. All rotational joints of the finger are connected via pivots with retaining rings at both sides, as shown in Fig. 2(b). The pivot 3D models are supplied with the gripper CAD model and can be used for manufacturing metal pivots using standard machine shop facilities.

\section{B. Gear Train Design}

The three fingers in the gripper are actuated by a single actuator using a gear train transmission system. The $2 \mathrm{D}$ and $3 \mathrm{D}$ representations of the single finger actuation is shown in Fig.3. A servo actuator is fixed on the base and drives the directly connected worm gear. The worm transfers rotational motion to the set of worm wheels, which are connected to each finger. All three fingers are driven simultaneously from their initial position. Usage of the worm gear ensures nonbackdriveability of the finger actuation mechanism. The set of two worm wheels are placed in a way that allows adjustment of the rotational speed and torque transferred to a finger by changing the gear ratios within the given distance between gear rotational axes. Researchers can also change the actuator and set up custom gear train speed/torque ratio depending on their need without modifying the overall gripper design.

As rapid prototyping technology can impose limitations in producing strong and precise miniature parts due to the low resolution and/or printing material properties of $3 \mathrm{D}$ printers, the gear train design utilizes a minimal number of small size parts. The presented worm and the worm wheels are designed to allow easy 3D printing that is achieved with high gear tooth module number. So one can easily replace to their own different variants depending on custom gripper requirements. One should take note that the layer resolution of used $3 \mathrm{D}$ printer is up to $0.15 \mathrm{~mm}$ [21]. It is not possible to create small and miniature gears with small teeth for the gear train. More advanced and sophisticated 3D printer can be used instead.

\section{Three Fingered Robotic Gripper}

The three fingered robotic gripper consists of the three 2DOFs underactuated fingers, a base, a palm, a gear train set and an actuator. The fingers are attached in a circular way with 120 degrees between each other. This allows the worm wheels of the fingers to be driven from a single actuator via the actuating worm as illustrated in Fig. 4. This type of layout of the fingers is preferable for grasping spherical or cylindrical objects of different sizes.

The CAD model of the robotic gripper and a $3 \mathrm{D}$ printed assembled prototype are presented in Fig. 5. The prototype is manufactured using 3D printing technology with ABS plastic and rubber materials. ABS is a strong, durable productiongrade thermoplastic used across many industries, and it is an ideal material for conceptual prototyping [22]. An additional off-the-shelf component, a Dynamixel MX-28 servomotor, is used as an actuator for robotic gripper prototype that eliminates necessary for complex electronic circuits and encoders for motor position control. Control of the actuator can be performed directly using MATLAB [23], a C/C++ programming environment and/or the Robot Operating System (ROS) [24], that provide easy and straightforward integration of the gripper with other robotic research setups. The output torque of Dynamixel MX-28 servomotor at $12 \mathrm{~V}$ is $2.5 \mathrm{Nm}$ [25].

The exploded assembly view of the gripper design is presented in Fig. 6. The actuator is mounted using bolts 


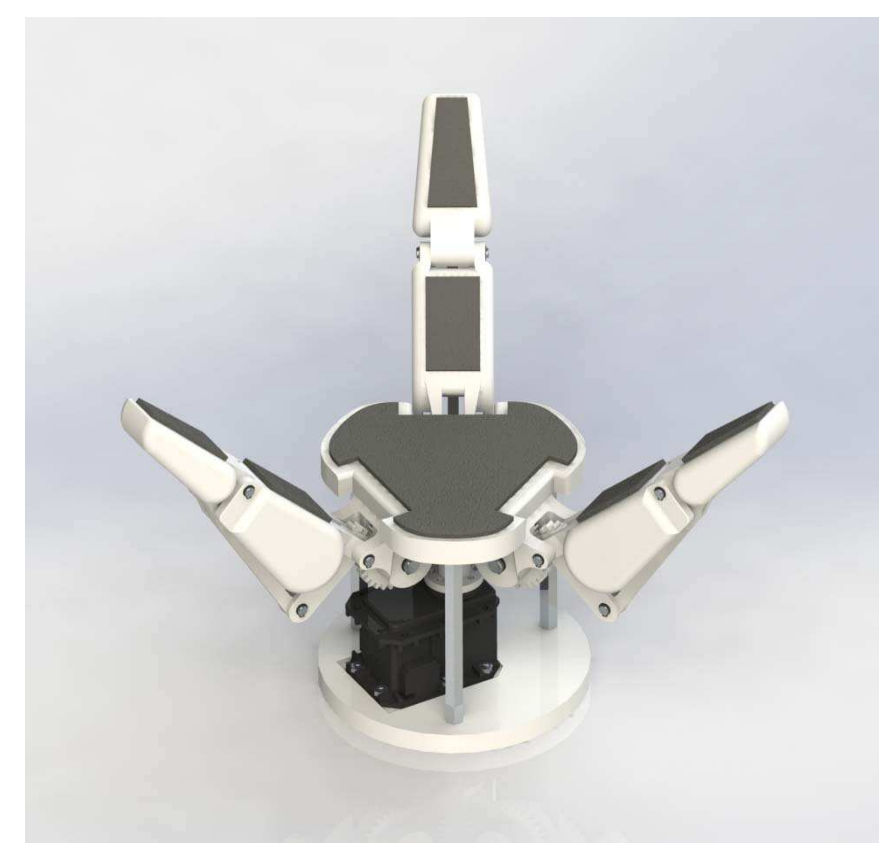

(a)

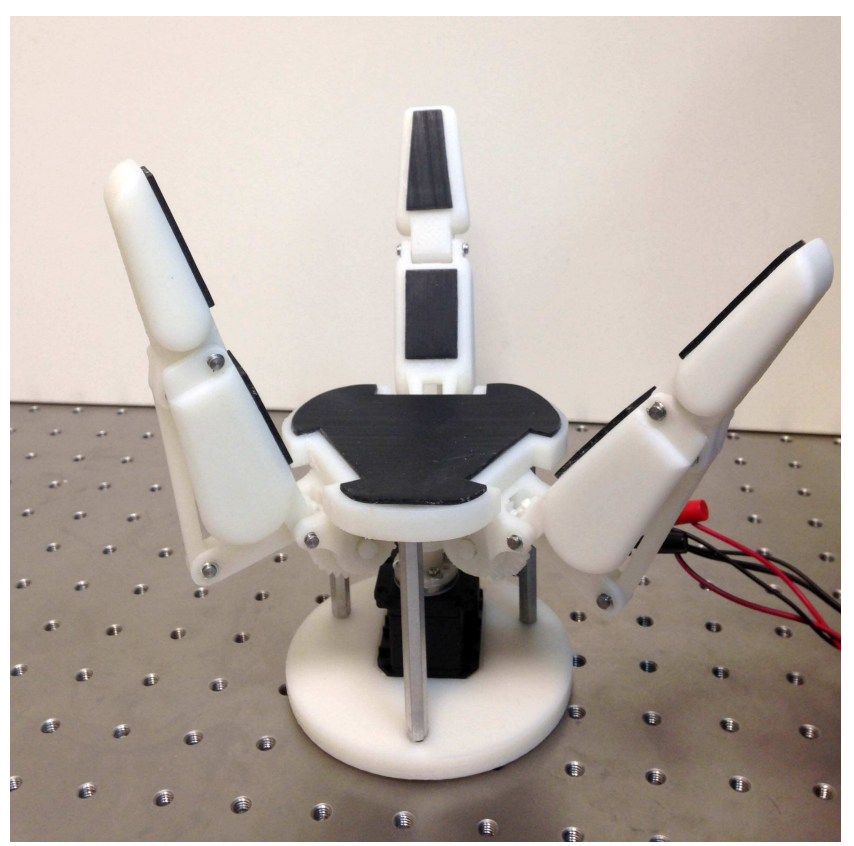

(b)

Fig. 5. Three fingered gripper CAD assembly model (a) and a 3D printed prototype (b).

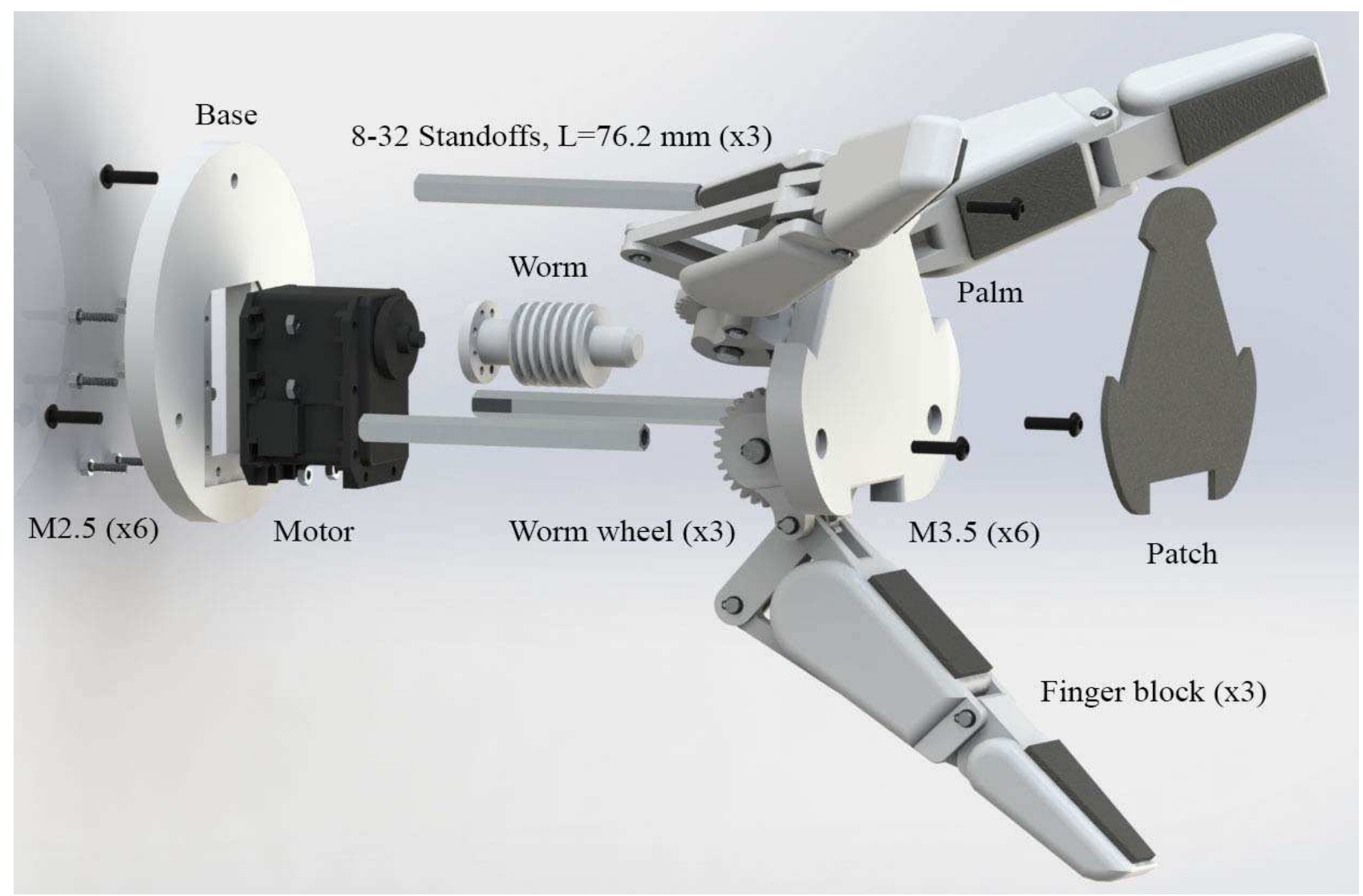

Fig. 6. Exploded assembly view of the presented three fingered gripper. 

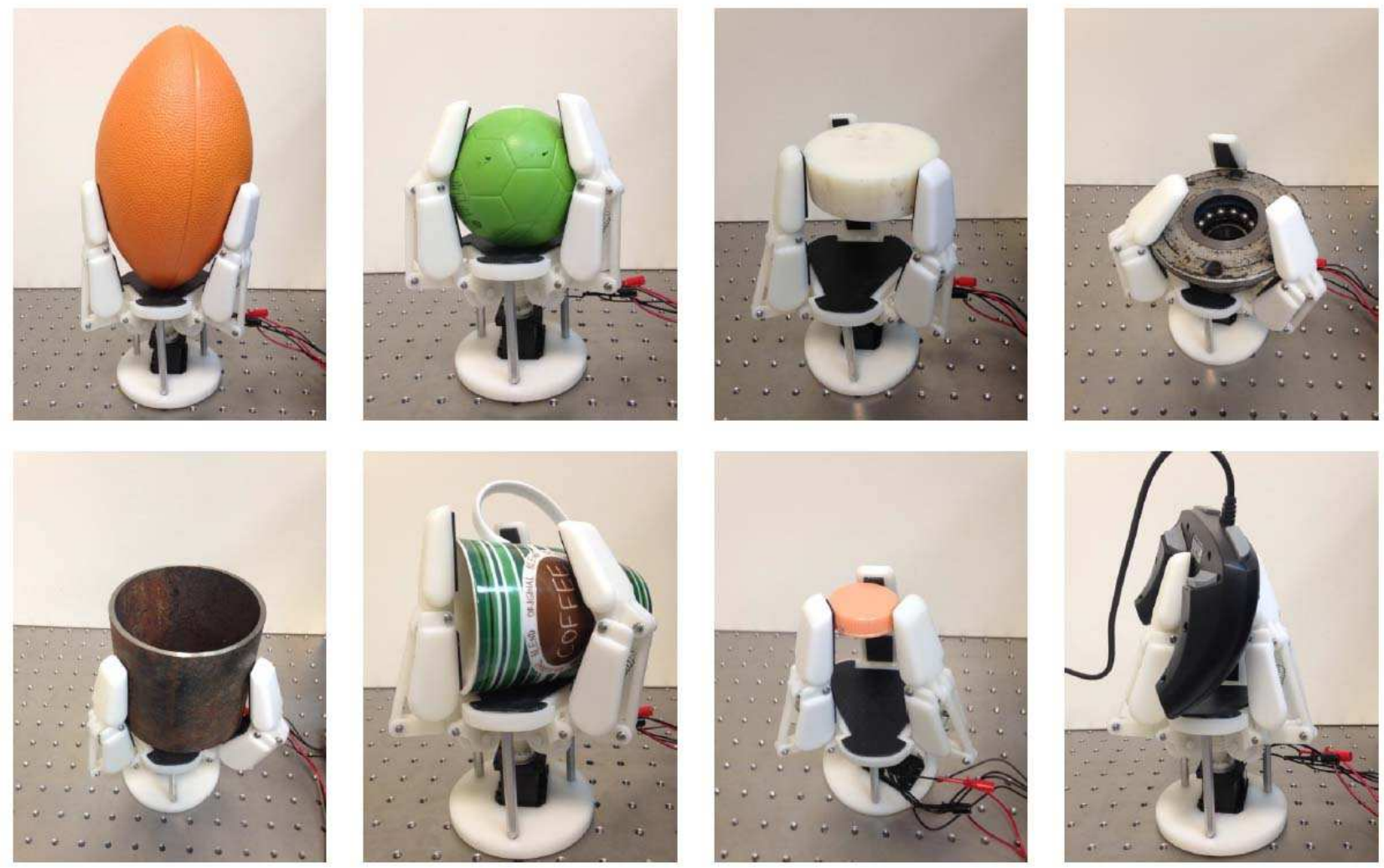

Fig. 7. Grasping of different objects using the presented three fingered gripper.

on a base with a circular shape and flat bottom. The base shape and attachment holes for mounting the gripper can be further created in a CAD program or easily drilled depending on robotic arm used. The worm is fixed to the actuator's output shaft at one side and to the palm at the other end. The worm axis is coincident with the symmetry axis of the gripper palm. The three fingers are fixed to the palm via pivots (shafts) with retaining rings and rotate about their pivot points. The palm and the base are connected to each other via three aluminium standoffs fixed by bolts from both sides. The standoffs with various length can be used to accommodate alternative gear trains. The palm and the fingers have spacings for thin rubber cushions. These outer cushions are designed for reducing slippery between the gripper and a grasping object and can be 3D printed using any rubber-like material [26]. If needed, the cushions can be replaced by tactile sensors with minor modification of the finger and palm designs. The overall weight of the gripper with current configuration is 500 grams.

\section{GRASPING PERFORMANCE}

The effective grasping of objects with different shapes and sizes is one of the important gripper design requirements. The presented underactuated gripper prototype ensures adaptive passive grasping and full wrapping of grasping objects. Fig.7 illustrates the performance of the robotic gripper in grasping a number of objects with different shapes. As shown in the figure during the grasping of an egg shaped ball all the three gripper fingers and their phalanges move as single rigid bodies and their passive elements are not active.

On the other hand, the springs in each finger provide the second DOF when grasping a spherical shaped ball: as all the first phalanges of the fingers touch the ball, the second phalanges envelop the object and complete grasping. The same grasping pattern is observed in grasping short cylindrical objects such as a bearing block.

Small objects are normally grasped by second phalanges or finger tips only, performing the spherical pinch grasp.

Executing grasping of complex shape objects with strong asymmetric geometry, all the three fingers wrap of the object with different degree of bending depending on the object shape. For example, in the case of grasping of a cup and a joystick objects, one finger moves as a single rigid body while the others actuate the second DOF and envelop the object. Thus, it is shown the gripper can successfully grasp objects with different shapes and sizes and can used with further customisation for research and educational purposes.

The maximum gripping force at the tip of the robotic gripper prototype was experimentally estimated as $9 \mathrm{~N}$. However, due to non-backdriveability of the gripper actuating mechanism, the gripper fingers may resist much larger forces that they 
actually exert [27].

\section{CONCLUSION}

This paper presents authors' design of an open source 3D printed underactuated robotic gripper. The gripper design model and an experimental prototype are introduced and discussed in detail. It is shown that the the presented robotic gripper with one actuator meets design objectives in terms of:

- a simple mechanical structure of the gripper due to usage of a four-bar linkage mechanical system for finger design and a single actuation;

- a low cost due to utilizing of a single off-the-shelf actuator and 3D printing technologies;

- relatively high payload comparable with similar size tendon driven robotic end effectors.

The 3D model of the gripper is created using the SolidWorks CAD software and will be available for downloading from the authors research lab website www.alaris.kz/. Similar to [12], the open source gripper design aims to overcome constraint of the rigid, closed designs of existing robotic hands. The simple three fingered robotic gripper design released open-source for public use would allow researchers modifying the design for both research and educational purposes.

Future work includes design and implementation of the presented end effector prototype with embedded sensing elements such as tactile sensors for force feedback capabilities and a depth camera for object recognition to facilitate research on autonomous grasping of different shape objects. The gripper prototype will also be mounted on an industrial robotmanipulator for evaluating grasping performance in real-life scenarios.

\section{REFERENCES}

[1] "Shadow Dexterous Hand Technical Specification," 2013. [Online]. Available: http://www.shadowrobot.com/wpcontent/uploads/shadow_dexterous_hand_technical_specification E1_20130101.pdf

[2] S. C. Jacobsen, E. K. Iversen, D. Knutti, R. Johnson, and K. Biggers, "Design of the Utah/M.I.T. dextrous hand," in Proceedings of the 1986 IEEE International Conference on Robotics and Automation (ICRA), vol. 3, Apr 1986, pp. 1520-1532.

[3] M. Baril, T. Laliberté, C. Gosselin, and F. Routhier, "On the design of a mechanically programmable underactuated anthropomorphic prosthetic gripper," ASME Journal of Mechanical Design, vol. 135, no. 12, 2013.

[4] T. Laliberté, L. Birglen, and C. Gosselin, "Underactuation in robotic grasping hands," Machine Intelligence \& Robotic Control, vol. 4, no. 3, pp. 1-11, 2002.
[5] A. Namiki, Y. Imai, M. Ishikawa, and M. Kaneko, "Development of a high-speed multifingered hand system and its application to catching," in Proceedings of the 2003 IEEE/RSJ International Conference on Intelligent Robots and Systems (IROS), vol. 3, Oct 2003, pp. 2666-2671.

[6] "Servo-Electric 3-Finger Gripping Hand SDH." [Online]. Available: http://www.schunk.com/schunk files/attachments/SDH DE EN.pdf

[7] W. Townsend, "The Barret Hand Grasper - Programmably Flexible Part Handling and Assembly," Industrial Robot, vol. 27, pp. 181-188, 2000.

[8] "Three-Finger Adaptive Robot Gripper." [Online] Available: http://robotiq.com/media/Robotiq-3-Finger-Adaptive-RobotGripper-Specifications.pdf

[9] Kinova, "Jaco research edition." [Online]. Available: http://kinovarobotics.com/wp-content/uploads/2013/05/kinovajaco-spec-sheet.pdf

[10] Schunk, "Schunk 3-finger concentric gripper sgw." [Online]. Available: http://www.schunk.com/schunk_files/attachments/SGW_gesamt_EN.pdf

[11] Lacquey, "Lacquey Gripper A101." [Online]. Available: http://food.lacquey.nl/sites/default/files/sheets/ SpecificationSheetSphericalGripper.pdf

[12] R. R. Ma, L. U. Odhner, and A. M. Dollar, "A modular, open-source 3D printed underactuated hand," in Proceedings of the 2013 IEEE International Conference on Robotics and Automation (ICRA), 2013, pp. 2737-2743.

[13] Z. Kappassov, Y. Khassanov, A. Saudabayev, A. Shintemirov, and H. Varol, "Semi-anthropomorphic 3D printed multigrasp hand for industrial and service robots," in 2013 IEEE International Conference on Mechatronics and Automation, August 2013, pp. 1697-1702.

[14] C.-H. Chen, "Mechatronics design of multi-finger robot hand," in 12th International Conference on Control, Automation and Systems, October 2012, pp. 1491-1496.

[15] G. Carbone, Grasping in Robotics, ser. Mechanisms and Machine Science. Springer, 2012.

[16] T. Laliberte and C. M. Gosselin, "Underactuated mechanical finger with return actuation," US Patent Application Publication \# 6,505,870.

[17] L. J. C. L'Ecuyer and C. Dequire, "Mechanical finger," US Patent Application Publication \# 13/377,600, July 19, 2012. [Online]. Available: https://www.google.com/patents/US20120185061

[18] S. Montambault and C. M. Gosselin, "Analysis of underactuated mechanical grippers," ASME Journal of Mechanical Design, vol. 123, no. 3, pp. 367-374, 2001.

[19] L. Birglen and C. Gosselin, "Geometric design of three-phalanx underactuated fingers," ASME Journal of Mechanical Design, vol. 128, no. 2 pp. 356-364, 2005.

[20] G. Figliolini and P. Rea, "Ca.U.M.Ha. robotic hand (cassinounderactuated-multifinger-hand)," in 2007 IEEE/ASME international conference on Advanced Intelligent Mechatronics, Sept 2007, pp. 1-6.

[21] “UP! 3D Printer," http://www.pp3dp.com/.

[22] "Stratasys ABS Material Properties," http://www.nrri.umn.edu/NLTC/ABS07.pdf.

[23] "MATLAB," http://www.mathworks.com/products/matlab/.

[24] "The Robot Operating System (ROS)," http://www.ros.org/.

[25] "Dynamixel MX-28," http://support.robotis.com/en/product/ dynamixel/mx series/mx-28.htm.

[26] "Stratasys PolyJet Rubber-Like Materials," http://www.stratasys.com/ rmedia/Main/Secure/Material20Specs20MS/ PolyJet-Material-Specs/PolyJet_Materials_Data_Sheet.pdf.

[27] S. Bartholet, "Reconfigurable end effector," US Patent Application Publication \# 5,108,140, April 28, 1992. [Online]. Available: https://www.google.com/patents/US5108140 